\title{
Shadow graph studies of laser-assisted non-thermal structuring of thin layers on flexible substrates by shock-wave-induced delamination processes
}

\author{
P. Lorenz ${ }^{1}$, T. Smausz ${ }^{2,3}$, T. Csizmadia ${ }^{2}$, M. Ehrhardt ${ }^{1}$, K. Zimmer ${ }^{1}$, B. Hopp ${ }^{2}$ \\ ${ }^{1}$ Leibniz-Institut für Oberflächenmodifizierung e. V., Permoserstraße 15, 04318 Leipzig, Germany \\ ${ }^{2}$ Department of Optics and Quantum Electronics, University of Szeged, H-6720 Szeged, Dóm tér 9, Hungary \\ ${ }^{3}$ MTA-SZTE Research Group on Photoacoustic Spectroscopy, University of Szeged, H-6720 Szeged, Dóm tér 9, \\ Hungary
}

\begin{abstract}
The laser-assisted microstructuring of thin films especially for electronic applications without damaging the layers or the substrates is a challenge for the laser micromachining techniques. The laser-induced thin-film patterning by ablation of the polymer substrate at the rear side that is called 'SWIFD' - shock-wave-induced film delamination patterning has been demonstrated. This study focusses on the temporal sequence of processes that characterize the mechanism of this SWIFD process on a copper indium gallium selenide (CIGS) solar cell stacks on polyimide. For this purpose high-speed shadowgraph experiments were performed in a pump probe experimental set-up using a $\mathrm{KrF}$ excimer laser for ablating the rear side of the polyimide substrate and measuring the shock wave generation at laser ablation of the polymer substrate as well as the thin-film delamination. The morphology and size of the thin-film structures were studied by scanning electron (SEM). Furthermore, the composition after the laser treatment was analyzed by energy dispersive X-ray spectroscopy (EDX). The shadowgraph experiments allow the time-dependent identification and evaluation of the shock wave formation, substrate bending, and delamination of the thin film in dependence on the laser parameters. These results will contribute to improve the physical understanding of the laser-induced delamination effect for thin-film patterning.
\end{abstract}

Keywords: excimer laser, structuring, delamination, CIGS, solar cell, SWIFD

\section{Introduction}

The conversion of the energy supply to renewable energy sources represents one of the most important and necessary industrial challenges where solar cells can be play an important role if the fabrication of low-cost, high-efficient and robust solar cells can be improved. For example solar cell on flexible substrates allows the implementation in new applications fields where the laser-based fabrication techniques exhibit an outstanding potential for the fast and easy structuring of thin layers especially on flexible substrates. However, the high thermal sensitivity of the active layer of the solar cell especially from organic semiconductors needs special requirements on the laser method. The direct ablation process for the selective structuring of the solar cell allows the fast patterning of the solar cell especially with ultra-short laser pulses [1], however secondary effects like thermal effects, ripples formation, and debris deposition processes restricted the practical applicability. The indirect laser methods like laser-induced shock waves structuring [2] can be applied for thin-film structuring with a distinct reduction of nuisance secondary effects. This method is called SWIFD (shock wave-induced film delamination) [2]. At SWIFD, the irradiation of the rear side of the substrate induced laser ablation and shock wave formation process, respectively and the mechanical effect (shockwave and bending of the substrate) on the front side result in a delamination effect of thin layers where the laser-induced shock wave in solids is well known [3]. Furthermore, the delamination effect can be discussed in relation to thermal-induced stress effects [4]. This patterning process can be used e.g. for CIGS solar cells [2]. The laser-induced shock wave effect can be used e.g. for the measurement of the adhesion of thin films [5] and for the laser-induced forward transfer (LIFT) of layer systems [6-8]. This study focus on the investigation of the laser induced secondary non-thermal processes that must be considered for the discussion of the SWIFD-mechanism by visualization of the shock and forces induced effects. Therefore, the time-resolved shadowgraph measurement that is regularly applied for laser driven mechanical processes [9-12] was used for the examining of the delamination. 


\section{Experimental set-up}

The primer experimental set up in Figure 1 shows a typical set-up for time resolved shadowgraph photography. For pumping the processes of SWIFD a KrF excimer laser is used that ablates polyimide easily. For imaging the beam of a dye laser illuminates the sample from the side in orthogonal configuration.

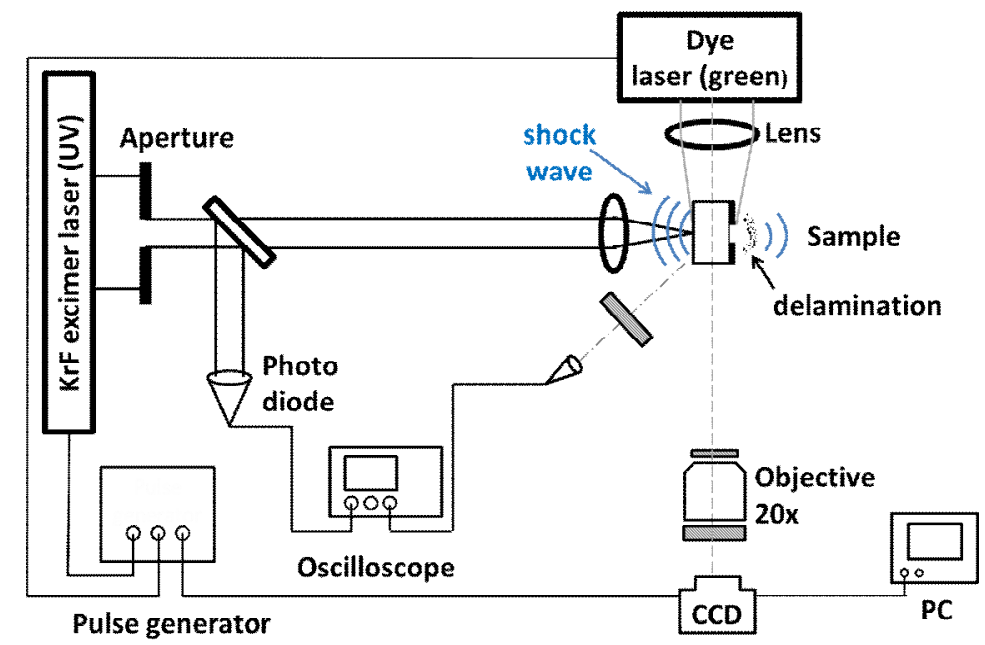

Fig. 1 Schematic illustration of the used pump probe experiment for time-resolved imaging

For the experiments $25 \mu \mathrm{m}$ thin polyimide foils covered with a full CIGS solar cell thin-film stack was used. The CIGS solar cell thin films include a indium tin oxide (ITO) front contact and a copper indium gallium selenide (CIGS) film with a entire thickness of $2.2 \mu \mathrm{m}$ as well as and a $1 \mu \mathrm{m}$ thick molybdenum (Mo) back. From the material samples were cut by scissors and clamped mechanically. Before the experiments the samples were adjusted to the imaging plane of the $\mathrm{CCD}$ camera. The rear side of the polyimide foil was irradiated by a $\mathrm{KrF}$ excimer laser pulses of a wavelength of $\lambda=248 \mathrm{~nm}$ and a pulse duration of $t_{p}=25 \mathrm{~ns}$. The focal spot size was approximately $550 \mu \mathrm{m}$. Furthermore, the laser-induced delamination effect was studied by time-dependent optical imaging of the side of the irradiated CIGS solar cell layer system where the sample was irradiated by an electronically delayed $\mathrm{N}_{2}$ laser pumped Coumarin 153 green dye laser $\left(\lambda=543 \mathrm{~nm}, \mathrm{t}_{\mathrm{p}}=1 \mathrm{~ns}, \mathrm{E}_{\mathrm{p}}<1 \mathrm{~mJ}\right)$. The side view of the layer system was imaged by a $20 \mathrm{x}$ objective with optical correction on an external triggered CCD image sensor. The time delay $(\Delta \tau)$ between the shock wave induced excimer laser, the illumination dye laser and the recording point of the camera could be adjusted by a freely programmable pulse generator. The delay between the excimer laser and the dye laser as well as the laser fluence stability of the excimer layer was controlled by photo diodes with a delay time of $1 \mathrm{~ns}$ where the diode signals were recorded by a $1 \mathrm{GHz}$ oscilloscope. After the shadowgraph experiments the laser irradiated sample surfaces were imaged by scanning electron microscopy (SEM) and their atomic composition was studied by energy-dispersive X-ray spectroscopy (EDX).

\section{Results and discussion}

The samples were irradiated at a laser fluence of $\Phi \approx 2.8 \mathrm{~J} / \mathrm{cm}^{2}$ and the images were recorded at different time delays. Except for pulse number studies at fixed laser parameters for each set of experimental properties a fresh not irradiated sample area was used. Hence typically only first pulse images are taken. Shadowgraph images at different time delays after the irradiation with a $\mathrm{KrF}$ excimer laser pulse with laser fluence of $\Phi=(2.8 \pm$ $0.15) \mathrm{J} / \mathrm{cm}^{2}$ are shown in Fig. 2. The images presented manifold laser-induced effects that influence the finally observed thin film patterning. The formation of a shock wave in the air near the laser-irradiated rear side of the sample can be observed at small time delays $(\Delta \tau<200 \mathrm{~ns})$. With increasing time delay ( $\Delta \tau \geq 500 \mathrm{~ns})$ the bending of the complete sample and the removal of material from the rear side of the PI substrate can be found. Finally, for even higher $\Delta \tau \geq 750 \mathrm{~ns}$ the thin film delamination and the fly away of the delaminated material can be seen. Further features that can be observed are shock wave formation near the front side of the sample for after $200 \mathrm{~ns}$ and bending of the flexible sample. The shadowgraph images are taken orthogonal to the samples 
surface and provide averaged information on the irradiated spot size or the depth of focus of the imaging 20x objective. Both surfaces of the sample, the front side with the film and the laser irradiated rear side are imaged after taking the shadowgraph series. The front side surface structures after the thin film delamination was measured by SEM and EDX. The SEM and EDX images of Fig. 3 show clearly the selective removal of the TCO/CIGS films from the Mo back contact without any signs of thermal impact or melting.
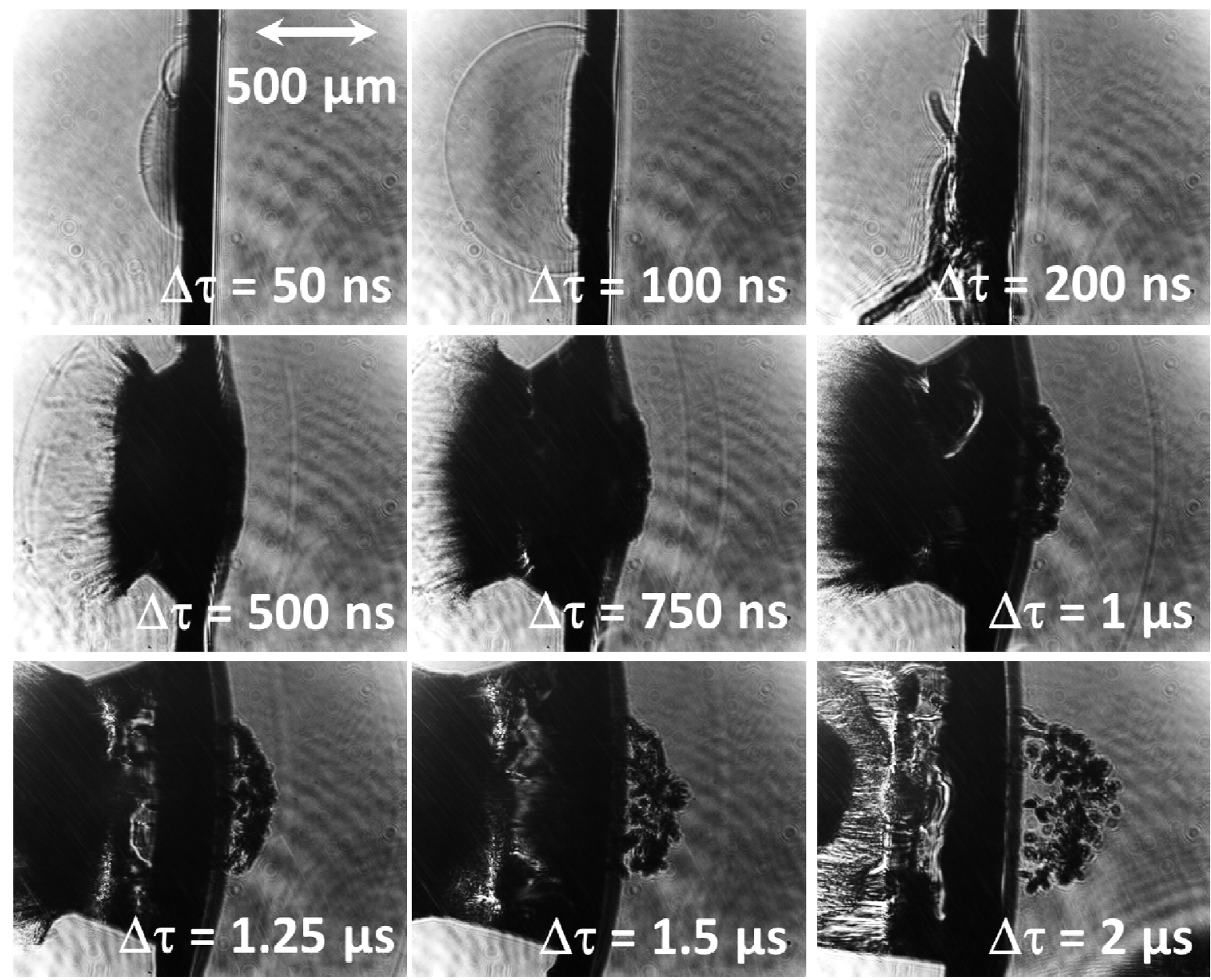

Fig. 2 Shadowgraph images of laser irradiated samples $\left(\Phi \approx 2.8 \mathrm{~J} / \mathrm{cm}^{2}\right)$ at different time delays $\Delta \tau$. The laser beam is focused on the rear side of the PI substrate, left side in the images.

As the surface analyzing shows the CIGS material is removed from the molybdenum back contact inhomogeneously with random distribution of angular shaped holes in the film whereas the percentage in the laser spot center is higher. The modified area has an average size of $\sim 400 \mu \mathrm{m}$ (area $\mathrm{A} \approx 0.13 \mathrm{~mm}^{2}$ ). These angular shaped holes in the CIGS film suggest that the film comes off in flakes. Further, the holes in the CIGS film are characterized by vertical, well defined edges (see Fig. 3 b). Both characteristics does not support a laser ablation mechanism but rather confirm the proposed shock wave induced delamination process [2]. Furthermore, the analysis of the chemical composition of the exposed Mo back contact presents that almost no residues of the CIGS can be found and the removal must be happen at the interface to the Mo film (see Fig. $3 \mathrm{c}$ and d). High resolution imaging of the exposed Mo film reveal no defects like cracks on the surface (see Fig. 3b). 


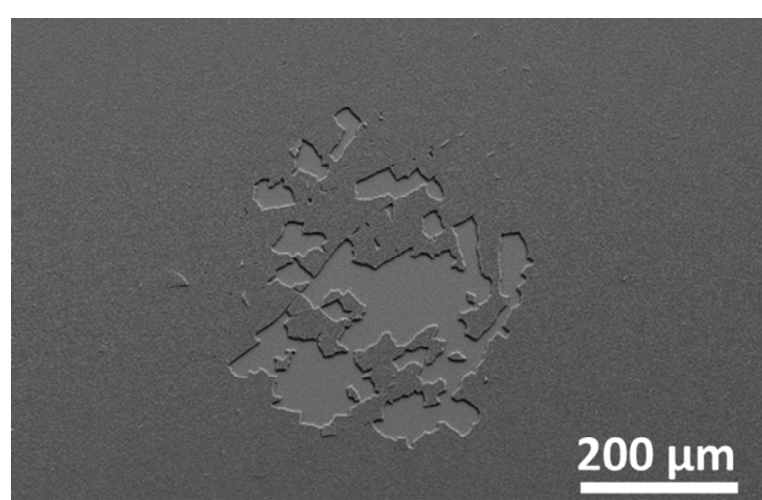

(a)

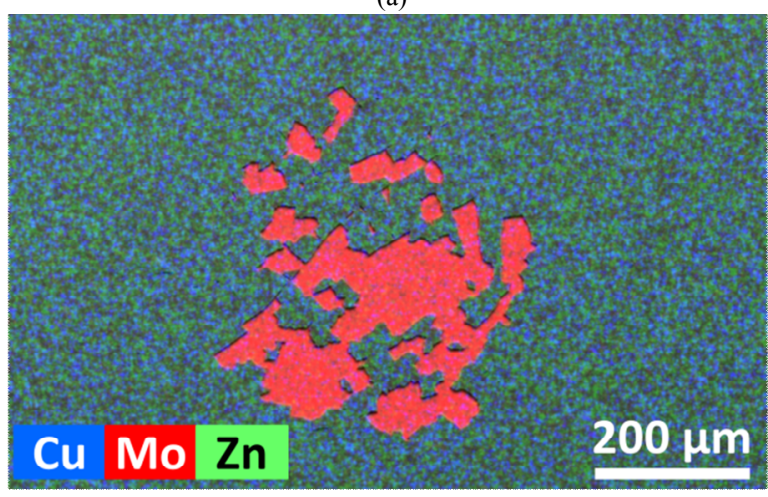

(c)

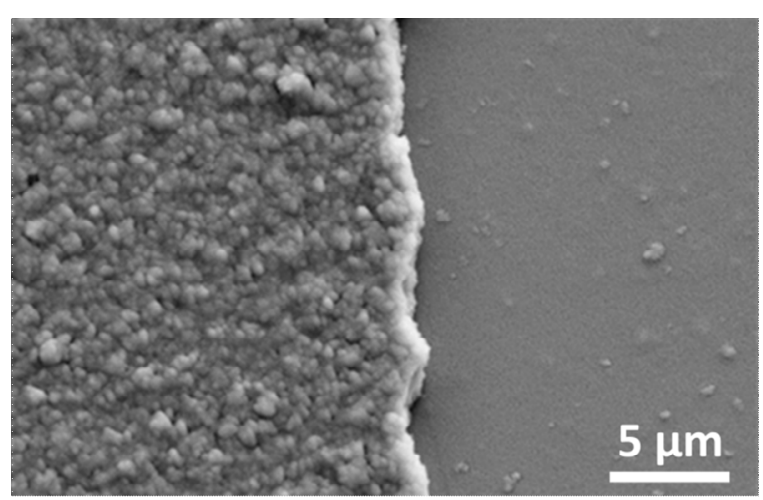

(b)

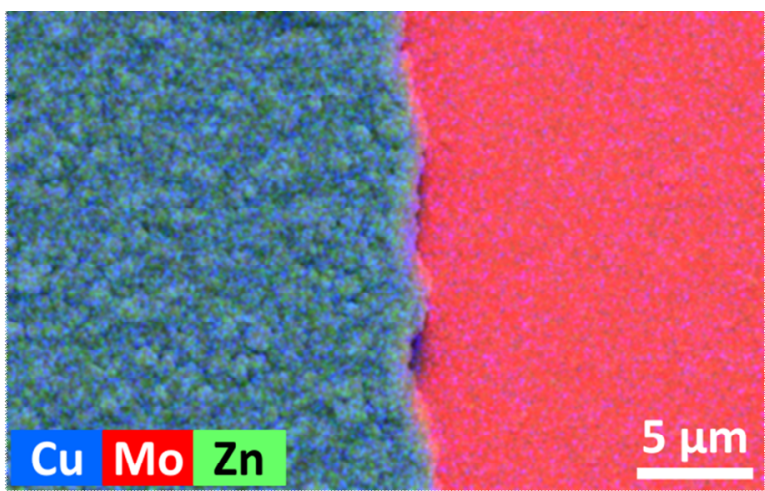

(d)

Fig. 3 SEM (a) and (b) and EDX images (c) and (d) after the laser treatment $\left(\Phi \approx 2.8 \mathrm{~J} / \mathrm{cm}^{2}, N=1\right)$

The different effects suggested by the shadowgraph measurements and the surface investigations are:

i. rear side shock wave formation process

ii. removal of material from the laser-irradiated side of PI substrate by ablation

iii. bending of the substrate

iv. front side shock wave formation

v. delamination and "fly away" of the film (CIGS) material

The different effects are schematically sketched in Fig. 4 without considering the time domain. (Note: In the sketch the expected effects summarizes despite the different time of occurrence.) 


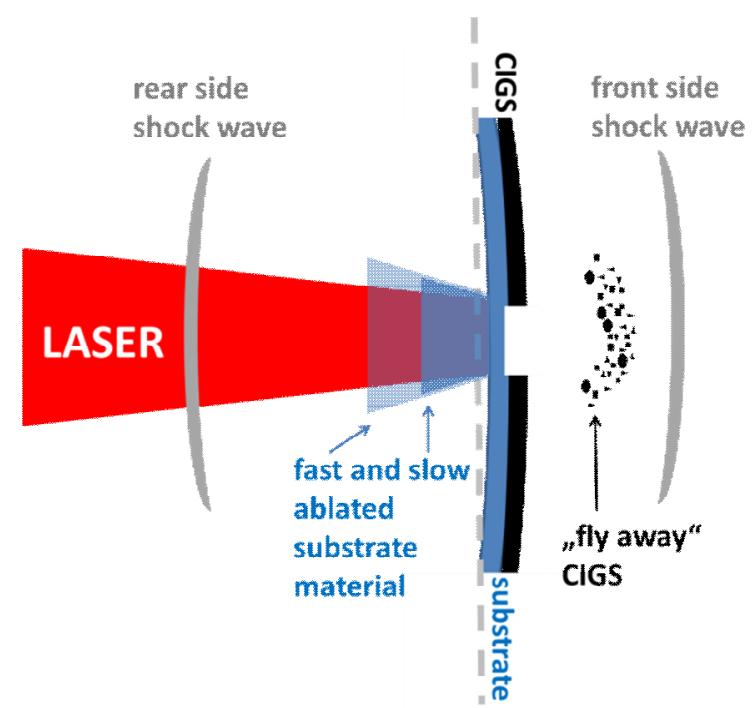

Fig. 4 Summarizing schematic illustration of the different feature observed by the shadowgraph measurements Note that the time domain of their occurrence is not considered in the sketch.

i. rear side shock wave formation process

The irradiation of the rear side of the substrate with a sufficient laser fluence result in the formation of a shock wave. The shock wave formation and the expansion of the shock wave is well visible in shadowgraph images (see Fig. 3 hemicycle feature on the left/rear side of the substrate at $\Delta \tau=50$ and $100 \mathrm{~ns}$ ). The shadow graph measurements show that the shock wave radius increases with time. The detailed analysis shows a decreasing shock wave velocity with increasing expansion time. In Fig. 5 (a) the maximal distance of the shock wave from the rear side of the substrate and the calculated velocity at different laser fluences are summarized. A slightly increase of the velocity at higher laser fluences can be seen.

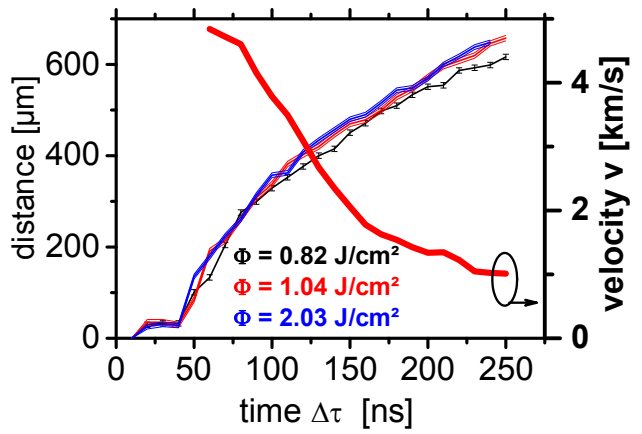

(a)

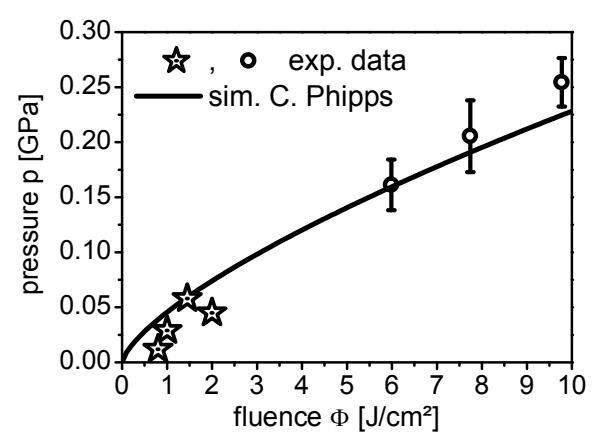

(b)

Fig. 5 (a) Distance of ablation-induced shock wave on the rear side of the PI substrate in dependence on time for different laser fluences and the calculated shock wave velocity for $\Phi=1.04 \mathrm{~J} / \mathrm{cm}^{2}$.

(b) pressure dependence on the laser fluence (stars: calculated from the shock wave velocity, circle: measured by a piezoelectric system, black line: $p(\Phi)$ dependency calculated by model of Phipps [13])

From the velocity dependence the maximum velocity at the surface can be estimated by extrapolation. With the maximum achievable shock wave velocity the pressure at the rear side can be estimated by [14]: 


$$
p(v)=\left(\frac{2 \cdot \rho_{g} \cdot v^{2}}{\gamma+1}\right) /\left(\frac{\gamma+1}{2 \gamma}\right)^{\frac{2 \gamma}{\gamma-1}} \underset{\substack{\gamma=1.4 \\ \rho_{g}=1.1839 \mathrm{~kg} / \mathrm{m}^{3}}}{\approx} 3.4172 \frac{P a \cdot s^{2}}{m^{2}} v^{2}
$$

Further, the pressure form laser ablation at particular conditions can be described by the model of C.R. Phipps [13]. The ablation pressure is dependent on the laser parameter like wave length, pulse duration, and laser fluence $\Phi$. It results for the applied laser:

$$
p(\Phi) \approx 37.8 \frac{\mathrm{MPa} \cdot \mathrm{cm}^{1.4}}{J^{0.7}} \cdot \Phi^{0.7}
$$

In Fig. 5 (b) the laser ablation pressure calculated from the shock wave velocities with Eq. 1 are compared with the estimated pressure by Phipps (Eq. 2) and direct measured pressures with a piezoelectric transducer according to [15-17]. The model of Phipps allows a good description of the shock wave pressure as seen from Fig. 5 (b). However, at low laser fluences a distinct deviation between the Phipps model at the calculated pressure from the shock wave velocity was found. Reasons therefore might be the neglection of the ablation threshold in the Phipps model or the change of the shock wave shape with expansion.

ii. removal of material from the rear side of PI substrate

Beside the shock wave formation the removal of the material from the rear side can be detected by the shadowgraph measurement. The speed of the ablated material is smaller than the shock wave velocity. In principle, the "fly away" material can be split in a slow part, well-visible black region on the left side of the rear side of the substrate, and a fast part, slightly visible undefined black and grey region above the "slow away" material (see Fig. $2, \Delta \tau=500 \mathrm{~ns}$ ). For the fast ablated material an average velocity of $\sim 1.5 \mathrm{~km} / \mathrm{s}$ can be estimated. From the distance of the "slow fly away" material in dependent on the time seen in Fig. 6 the velocity can be calculated.

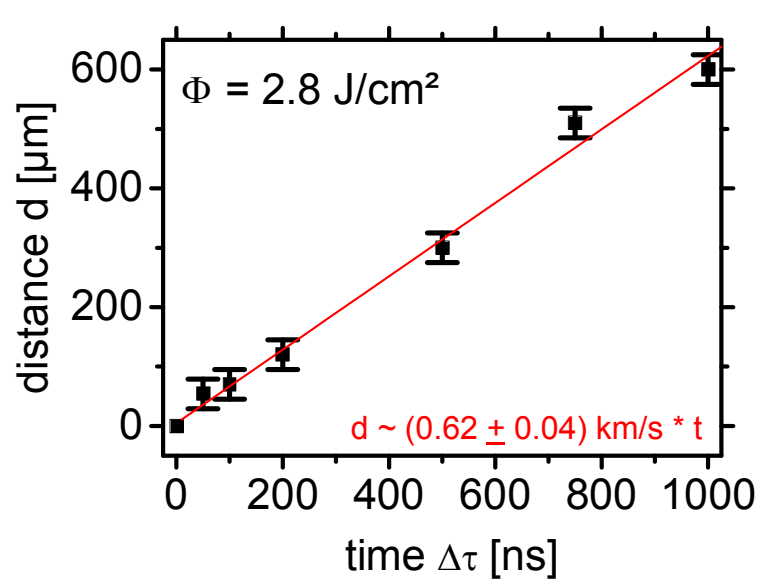

Fig. 6 Distance of the angular point of the "slow fly away" material from the rear side of the PI substrate at a laser fluence of $2.8 \mathrm{~J} / \mathrm{cm}{ }^{2}$ (black square: measured values, red line: linear plot)

Considering the experimental errors the experimental values can be fitted well with a linear function resulting in an average velocity of $(0.62 \pm 0.04) \mathrm{km} / \mathrm{s}$. An equal behavior separation in a slow and fast part was also found for the laser ablation of graphite with comparable velocities [18]. 
The laser-induced ablation pressure results in the bending of the substrate as it is visible in the shadow graph images. The identification of the bending of the front side of the CIGS solar cell is difficult especially at small time delays due to the overlap of bending and material removal seen as "fly away" CIGS material. In Fig. 7 the displacement $\Delta z$ of the bended front side of the sample to the initial position of the CIGS solar cell is plotted on the time.

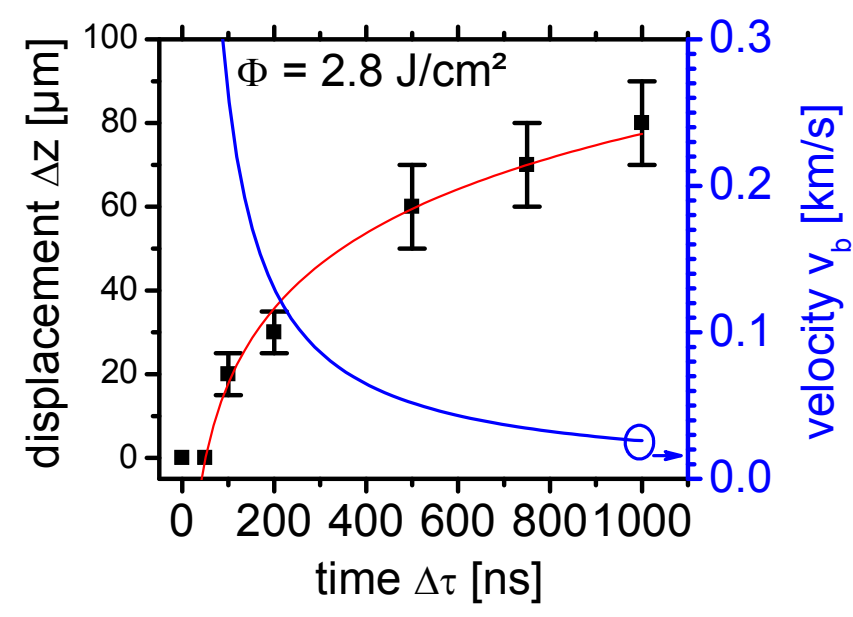

Fig. 7 Displacement $\Delta z$ depending on the time at $\Phi=2.8 \mathrm{~J} / \mathrm{cm}^{2}$ : black square: measured values, red line: logarithmic plot of the experimental values with $\Delta z(\Delta \tau)=b^{*} \ln \left(\Delta \tau+t_{0}\right)-a\left(a=(102 \pm 27) \mathrm{km} / \mathrm{s}, b=(26 \pm 4) \mathrm{km} / \mathrm{s}, t_{0} \approx 0\right.$; coefficient of determination $\left.R^{2}=0.991\right)$, blue line: speed of the lift off material $v_{b}$ (derivation of the logarithmic function $\Delta z(\Delta \tau)$ )

The experimental data can be well fitted by a logarithmic function with a coefficient of determination $\mathrm{R}^{2}$ of 0.991. From the fit function the velocity can be derived; the velocity of the lift off material in dependence on the time $\mathrm{v}_{\mathrm{b}}(\Delta \tau)$ is shown in Fig. 7 (blue line) too.

\section{iv. front side shock wave formation}

Beside the rear side shock wave formation in the air also a shock wave on the front side of the CIGS solar cell can be detected. The shock wave on the front side is delayed in comparison to the rear side shock wave. The first evidence for a shock wave at the front side can be detected after $200 \mathrm{~ns}$ for a fluence of $2.8 \mathrm{~J} / \mathrm{cm}^{2} \mathrm{but}$, the contrast of front side shock wave is distinguishably lower than the back side shock wave. For longer delay times multiple shock waves feature on the front side can be detected. From the measurement in the time range of 200 to $500 \mathrm{~ns}$ the average shock wave velocity was estimated to be $\sim 0.8 \mathrm{~km} / \mathrm{s}$. Hence, the order of magnitude of the rear and front side shock wave velocity is equal. In consequence, the shock wave features on the front side can be most likely explained by the $3 \mathrm{D}$ orbit of the shock wave around the CIGS solar cell originating from the rear side.

v. delamination and "fly away" of the CIGS material

The SEM and EDX images (see Fig. 3) presented that the laser irradiation induces a selective removal of the CIGS material from the molybdenum film. At higher delay times $\Delta \tau$ the flakes of and "fly away" CIGS material can be seen very well in the shadow graph images (see e.g. Fig. $2(\Delta \tau=2 \mu \mathrm{s})$ ). The measured distance of the lift off CIGS material from the initial position is shown in Fig. 8 (black squares). It has to be noticed that at low delay times the displacement of the CIGS is mainly defined by the bending of the substrate (see grey line: bending induced displacement). For delay times larger than $200 \mathrm{~ns}$ the lift off of the delaminated CIGS can be assumed. 


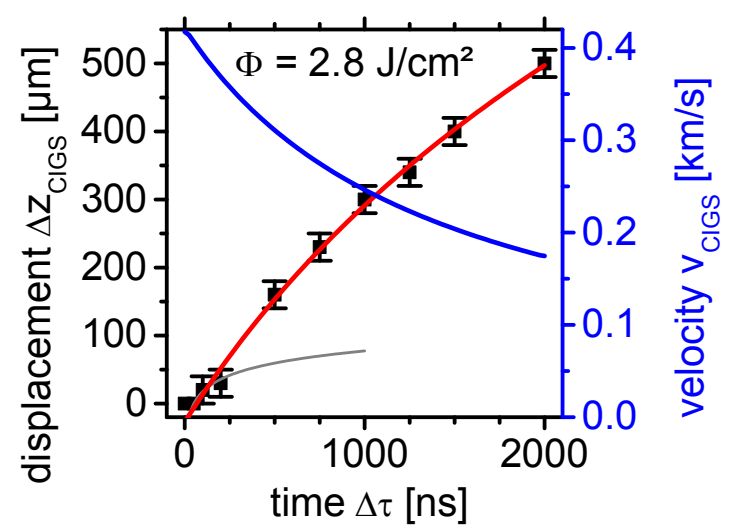

Fig. 8 Displacement of the CIGS $\Delta z_{C I G S}$ depend on the time at $\Phi=2.8 \mathrm{~J} / \mathrm{cm}^{2}$ : black square: measured values, red line: logarithmic plot of the experimental values with $\Delta z(\Delta \tau)=b^{*} \ln \left(\Delta \tau+t_{0}\right)-a\left(a=(4330 \pm 1040) \mathrm{km} / \mathrm{s}, b=(593 \pm 120) \mathrm{km} / \mathrm{s}, t_{0} \approx(1409 \pm 425) \mathrm{ns}\right)$; coefficient of determination $\left.R^{2}=0.9969\right)$, blue line: derivation of the logarithmic function equal to the velocity - time dependency $v_{b}(\Delta \tau)$, grey line: bending induced displacement

The distance of the lifted off CIGS material $\Delta \mathrm{z}_{\text {CIGS }}$ increase with the delay time and can be fitted very well by a logarithmic function (see red line Fig. 8; $\mathrm{R}^{2}=0.9969$ ). From the fitted analytic function the velocity of the "fly away" CIGS material was estimated. At the initial stage of the lift off a velocity of $\sim 0.4 \mathrm{~km} / \mathrm{s}$ can be found and after $\Delta \tau=2 \mu \mathrm{s}$ the velocity is reduced approximately by a factor of two. The extracted velocities agree with velocity found at LIFT processes using $\mathrm{KrF}$ excimer laser, whereas for LIFT smaller fluences are used that induce lower velocities [19]. The plotted displacements and velocities in Fig. 8 are maximum values characterizing the front side edge of the "fly away" CIGS. However, at high delay time a distinct variation of the displacement and velocity can be found, respectively. The most likely explanation for the widening of the blown off material are the error of this experimental setup, that images the 2D projection (CCD sensor) of the expected 3D expansion of the CIGS material. The images suggest that the displacement in the center of the irradiation is higher than at the border of the irradiation. Further, the speed of the CIGS flakes might be different across the laser spot due to the inhomogeneous fluence distribution and the local different bending speed of the substrate

\section{Conclusion}

The features recognized from the shadow graph images show clearly that the laser pulse energy is transferred into mechanical energy that finally results in the lift off of the thin film material that is here CIGS. Two reasons for lift off the CIGS material upon rear side ablation of the polyimide substrate have been identified that are the bending of the flexible substrate and the shock wave. The differentiation is not possible at the current state due to the insufficient lateral resolution of the images. In consequence further time resolved measurements are needed that focus on higher lateral resolution as well as on high resolution vertical imaging. The surface analytical results show that the process is of non-thermal nature. The steep side walls, the absence of melting signs and the angular shaped holes are clear indices therefore. This non-thermal mechanism suggests that the properties of the film materials as well as the characteristics of the thin film stack are not influenced by the patterning process. Hence, SWIFD of CIGS is a good alternative to the "classical" direct ablation of the material. Due to very complex mechanism the analytical description is challenging. Hence, FEM simulations will be performed.

\section{Acknowledgement}

The research leading to these results has received funding from the European Community's Seventh Framework Program (FP7/2007-2013) under APPOLO project (grant agreement No. 609355), the Deutsche Forschungsgemeinschaft (DFG) under contract ZI 660/12-1 and the German Academic Exchange Service (DAAD).

\section{References}

[1] Gečys P., "Ultrashort pulsed laser processing of thin films for solar cells" PhD thesis, Vilnius university (2012). 
[2] M. Ehrhardt et al., Appl. Phys. A 113, 309-313 (2013).

[3] Ding, K., Ye, L., "Laser Shock Peening: Performance and Process Simulation”, 1st edn., CRC Press, Boca Raton (2006).

[4] Bovatsek, J. et al., Thin Solid Films 518, 2897-2904 (2010).

[5] Wang, J.L. et al., Exp. Mech. 42, 74-83 (2002).

[6] Serra, P. et al., Cell and Organ Printing, 53-80 (2010).

[7] Ihlemann, J., Weichenhain-Schriever, R., Thin Solid Films 550, 521 - 524 (2014).

[8] Murphy, R.D. et al., Appl. Phys. Lett. 103, 093113 (2013).

[9] Zhang N. et al., Phys. Rev. Lett. 99, 167602 (2007).

[10] Yoo J. H. et al., J. Appl. Phys. 88, 1638 - 1649 (2000).

[11] Zeng, X. et al., Appl. Phys. A 80, 237 - 241 (2005).

[12] Mattle T. et al., Appl. Surf. Sci. 278, 77-81 (2013).

[13] Phipps, C.R., et al., J. Appl. Phys. 64, 1083-1096 (1988).

[14] Zel'dovichand Y. B., Raizer Y. P., "Physics of shock waves and high-temperature hydromanic phenomena", Dover Publications, Inc. Mineola, New York, 2002

[15] Zweig, A. D. et al., J. Appl. Phys. 7, 4181-4189 (1993).

[16] Olmes, A., et al., Appl. Phys. B 64, 677-682 (1997).

[17] Ehrhardt M., „Laserinduziertes nichtthermisches Hochgeschwindigkeitsumformen von Kupfer“, PhD Thesis University Leipzig (2014) (submitted).

[18] Young-Ku C., Bull. Korean Chem. Soc. 20, 1501-1505 (1999).

[19] Perinchery S. M., Laser Phys. 24, 066101 (2014). 